ARTICLE OPEN

\title{
Enhanced interlayer neutral excitons and trions in trilayer van der Waals heterostructures
}

\author{
Chanyeol Choi ${ }^{1,2,9}$, Jiahui Huang ${ }^{1,2}$, Hung-Chieh Cheng ${ }^{3,4}$, Hyunseok Kim $\mathbb{D}^{2}$, Abhinav Kumar Vinod ${ }^{1,2}$, Sang-Hoon Bae ${ }^{3,4,10}$, \\ V. Ongun Özçelik ${ }^{5}{ }^{5}$, Roberto Grassi ${ }^{6}$, Jongjae Chae ${ }^{3}$, Shu-Wei Huang ${ }^{1,2,11}$, Xiangfeng Duan ${ }^{4,7}$, Kristen Kaasbjerg $^{8}$, Tony Low $^{6}$ and \\ Chee Wei Wong ${ }^{1,2}$
}

Vertically stacked van der Waals heterostructures constitute a promising platform for providing tailored band alignment with enhanced excitonic systems. Here, we report observations of neutral and charged interlayer excitons in trilayer $\mathrm{WSe}_{2}-\mathrm{MoSe}_{2}-\mathrm{WSe}_{2}$ van der Waals heterostructures and their dynamics. The addition of a WSe $\mathrm{W}_{2}$ layer in the trilayer leads to significantly higher photoluminescence quantum yields and tunable spectral resonance compared to its bilayer heterostructures at cryogenic temperatures. The observed enhancement in the photoluminescence quantum yield is due to significantly larger electron-hole overlap and higher light absorbance in the trilayer heterostructure, supported via first-principles pseudopotential calculations based on spin-polarized density functional theory. We further uncover the temperature- and power-dependence, as well as timeresolved photoluminescence of the trilayer heterostructure interlayer neutral excitons and trions. Our study elucidates the prospects of manipulating light emission from interlayer excitons and designing atomic heterostructures from first-principles for optoelectronics.

npj 2D Materials and Applications (2018)2:30 ; doi:10.1038/s41699-018-0075-1

\section{INTRODUCTION}

In two-dimensional (2D) materials, Coulomb-induced electronic states of excitons have been examined as a platform to understand many-body carrier-carrier interactions. These excitonic interactions dominate in layered materials due to quantum confinement and reduced dielectric screening. 2D atomic crystals of transition metal dichalcogenides (TMDs) have provided new opportunities in the studies of single-exciton single-photon interactions, spin-orbit coupling, ultrafast dynamics, and nanoelectronic devices. ${ }^{1-22}$ Layer-by-layer stacking of TMDs-based van der Waals (vdWs) heterostructures has recently captured the attention of the scientific community where the tailored band alignment with diverse 2D materials can be achieved via advanced 2D growth and transfer techniques. ${ }^{23-28}$ This has allowed the extension of indirect excitons with spatially separated electrons and holes from, for example, coupled quantum wells in $\mathrm{GaAs} / \mathrm{AlGaAs}^{29,30}$ to TMD vdW heterostructure bilayers, where they are referred to as interlayer excitons. These interlayer excitons exhibit rich physics in TMDs-based vdWs heterostructures due to the novel atomic granularity control through layer-by-layer stacking and the chiral properties of quantum electronic states. ${ }^{8,31,32}$ Recently several studies observed that heterostructure interlayer excitons feature long lifetimes, spin-valley polarization by circularly-polarized pumping, near-unity valley polarization via large magnetic splitting, and charge transfer at the heterogeneous interfaces. ${ }^{32-45}$

Here, we demonstrate TMDs-based vdWs heterostructures composed of a three-layer $\mathrm{WSe}_{2}-\mathrm{MoSe}_{2}-\mathrm{WSe}_{2}$ stack to achieve unique band alignment that promotes efficient interlayer radiative recombination. We first design the heterostructure through pseudopotential calculations based on spin-polarized density functional theory (DFT), with the exchange-correlation potential estimated via the Perdew-Burke-Ernzerhof functional. Optimizing the designed heterostructure band structure through conjugate gradients, we include the effects of spin-orbit coupling through non-collinear computations. We correlate the obtained real-space pseudo-wavefunctions with lattice points in reciprocal space to understand the carrier localization, the type-II optical transition oscillator strength, and potential asymmetries in the electron and hole band profiles towards neutral interlayer excitons and threebody trion complexes. Enabled by the first-principle computations, we fabricate the trilayer heterostructure stack through hexagonal boron nitride-assisted transfer. Our dry transfer process allows a relatively undoped heterostructure and atomic smoothness at the interfaces. We perform cryogenic photoluminescence on the designed trilayer heterostructure along with time-resolved pulsed

\footnotetext{
${ }^{1}$ Fang Lu Mesoscopic Optics and Quantum Electronics Laboratory, University of California, Los Angeles, CA 90095, USA; ${ }^{2}$ Department of Electrical Engineering, University of California, Los Angeles, CA 90095, USA; ${ }^{3}$ Department of Materials Science and Engineering, University of California, Los Angeles, CA 90095, USA; ${ }^{4}$ California Nanosystems Institute, University of California, Los Angeles, CA 90095, USA; ${ }^{5}$ Andlinger Center for Energy and the Environment, Princeton University, Princeton, NJ 08544, USA; ${ }^{6}$ Department of Electrical and Computer Engineering, University of Minnesota, Minneapolis, MN 55455, USA; ${ }^{7}$ Department of Chemistry and Biochemistry, University of California, Los Angeles, CA 90095, USA and ${ }^{8}$ Center for Nanostructured Graphene (CNG), Department of Micro- and Nanotechnology (DTU Nanotech), Technical University of Denmark, DK-2800 Kgs. Lyngby, Denmark

Correspondence: Chanyeol Choi (cowellchoi@gmail.com) or Chee Wei Wong (cheewei.wong@ucla.edu)

${ }^{9}$ Present address: Department of Electrical Engineering and Computer Science, Massachusetts Institute of Technology, Cambridge, MA 02139, USA

${ }^{10}$ Present address: Department of Mechanical Engineering, Massachusetts Institute of Technology, Cambridge, MA 02139, USA

${ }^{11}$ Present address: Department of Electrical, Computer, and Energy Engineering, University of Colorado Boulder, Boulder, CO 80309, USA
}

Received: 24 January 2018 Revised: 12 July 2018 Accepted: 2 August 2018

Published online: 17 September 2018 
measurements via time-correlated single-photon counting, mapping the spectral, pump fluence, and bath temperature dependence of the interlayer excitons. We observe an order-ofmagnitude larger photoluminescence in the trilayer compared to bilayer heterostructures, due to the more distributed hybridized valance band state in the trilayer for increased oscillator strength, along with the formation of the neutral interlayer exciton. The additional $\mathrm{WSe}_{2}$ layer absorbance in the trilayer also contributes to the stronger photoluminescence compared with the bilayer. At $4 \mathrm{~K}$, we observe a spectrally-distinguishable trion formation. We determine that the energy splitting between the neutral exciton and trion is largely dependent on the pump fluence and photocarrier densities instead of the bath temperature. We observe that the trion mostly red-shifts while the neutral exciton stays consistent and extract a trion binding energy of approximately $27 \mathrm{meV}$ in our trilayer heterostructure. This result is close to the interlayer negative charged exciton binding energy of $28 \mathrm{meV}$ as predicted by the recent theoretical study on the vertical stack $\mathrm{MoS}_{2} / \mathrm{WS}_{2}$ heterostructures. ${ }^{46}$ With increasing photocarrier injection and decreasing bath temperatures, the trion-to-neutral intensity ratio is also increased. Radiative lifetimes of the interlayer excitons are measured up to $\approx 2.54 \mathrm{~ns}$ at $4 \mathrm{~K}$. We examine the radiative lifetimes for different bath temperatures, pump fluences, and excitation wavelengths, with observed stronger dependences on the bath temperature. Our study enables the engineering of indirect neutral excitons and charged trions with spatially separated electron and hole wavefunctions, towards control of the oscillator strengths and excitonic character with designed atomic granularity.

\section{RESULTS}

Theoretical analysis

Here, we introduce the concept towards the enhancement of the exciton recombination rate of the trilayer heterostructure illustrated in Fig. 1a, b. The trilayer heterostructure consists of a single-layer (SL) $\mathrm{MoSe}_{2}$ sandwiched between two SLs of $\mathrm{WSe}_{2}$. The anticipated type-II band alignment, depicted in Fig. 1b, supports excitons with the electron and hole residing respectively in the conduction band of $\mathrm{MoSe}_{2}$ and valence band of $\mathrm{WSe}_{2}$. This resembles the situation for excitons in heterostructure bilayers, where the electron and hole are strongly localized to the different layers by the large band offsets. ${ }^{47-49}$ This is indeed the case for the electron in the conduction band of the trilayer structure, which is strongly localized in the central $\mathrm{MoSe}_{2}$ layer due to a weak interlayer coupling. ${ }^{50}$ However, in our case, the valence band states are subject to a stronger interlayer coupling. ${ }^{50}$ Consequently, the valence band states in our two outer $\mathrm{WSe}_{2}$ layers hybridize with the state in the central $\mathrm{MoSe}_{2}$ layer. Accordingly, the delocalization of the valence band state over the entire trilayer enables the strong overlap of the electron-hole in the trilayer, compared to the prior bilayer counterparts.

To support this physical picture, we carried out first-principles DFT calculations including the spin-orbit interaction of the bilayer and trilayer band structures and wavefunctions, with the computational details described in the Methods. The results are summarized in Fig. 1c, d. (Band structures of SL $\mathrm{MoSe}_{2}$ and SL $\mathrm{WSe}_{2}$ are shown in Supplementary Section S1.) While the bilayer heterostructure shows $a \approx 1.22 \mathrm{eV}$ bandgap, the trilayer heterostructure has a slightly smaller $\approx 1.21 \mathrm{eV}$ bandgap, both at the Kpoint. In both the bilayer and trilayer structures, our calculations predict states which are predominantly localized in either $\mathrm{WSe}_{2}$ (blue) or $\mathrm{MoSe}_{2}$ (red) layers, in agreement with the band alignment illustrated in Fig. $1 \mathrm{~b}$. Figure $1 \mathrm{e}, \mathrm{f}$ shows the spatial distribution profile of the electron and hole probability densities in the out-of-plane direction, for the states at the valence and conduction band edges. In the bilayer, the electron and hole states hardly overlap, hence the long lifetime, ${ }^{31,32,51,52}$ and our weaker PL is shown in Figure S3. On the other hand, in the trilayer structure the valence band state shows the expected delocalization with a large center-layer component and thus a significant overlap with the localized conduction band state.

In our proposed scheme as shown in Fig. 1b, the type-II staggered heterojunction leads to energetically lower excitonic states due to the band offset between $\mathrm{MoSe}_{2}$ and $\mathrm{WSe}_{2}$. Furthermore, with the trilayer heterostructure, the strong asymmetry between the electron and hole band profile promotes excitons complexes such as trions. ${ }^{2,16,33,39,53}$ Unlike bilayer TMD heterostructures, a metallic state through biasing the Fermi level in the conduction or valence band is required in the trilayers to produce imbalance in electron and hole populations and hence increase the likelihood of trions formation. ${ }^{33,39}$ The interlayer trions can be achieved, for example, with two electrons in the $\mathrm{MoSe}_{2}$ layers and one hole in the WSe $\mathrm{W}_{2}$ layer. A description of the radiative recombination pathways in the trilayer and the trions is shown in Supplementary Section S2.

\section{Trilayer sample preparation and cryogenic photoluminescence} spectroscopy

We fabricate the $\mathrm{WSe}_{2}-\mathrm{MoSe}_{2}-\mathrm{WSe}_{2}$ trilayer heterostructure on a $\mathrm{SiO}_{2} / \mathrm{Si}$ substrate by dry transfer with a hexagonal boron nitride $(h-$ $\mathrm{BN}$ ) flake of $20 \mathrm{~nm}$ thickness as shown in Fig. 2a. Both SL WSe 2 and $\mathrm{SL} \mathrm{MoSe}_{2}$ are mechanically exfoliated from bulk crystals and identified with optical microscopy, $\mathrm{PL}$, and Raman spectra. Before the stacking process, the sample thickness is further confirmed independently via atomic force microscopy (AFM) as shown in Fig. 2b. Raman spectroscopy is conducted on the $\mathrm{SL} \mathrm{MoSe}{ }_{2}, \mathrm{SL} \mathrm{WSe} \mathrm{H}_{2}$ $20 \mathrm{~nm}$-thick $h$-BN as well as TMDs trilayer heterostructures as illustrated in Fig. 2c. In the inset of Fig. 2c, we observe two distinguishable modes at $243 \mathrm{~cm}^{-1}\left(\mathrm{MoSe}_{2}\right)$ and $247 \mathrm{~cm}^{-1}\left(\mathrm{WSe}_{2}\right)$. $20 \mathrm{~nm}$-thick $h$-BN is used as an encapsulating layer due to its atomic smoothness and relative absence of charge traps and dangling bonds. ${ }^{54}$ Raman shift of $h$-BN shows up in the TMDsbased trilayer heterostructure. The completed $h$-BN/WSe $/ \mathrm{MoSe}_{2} /$ $\mathrm{WSe}_{2}$ stacked structure is on a $290 \mathrm{~nm} \mathrm{SiO}{ }_{2}$ substrate. To avoid undesired doping, we do not use any chemicals. With regards to the stacking geometry, we randomly stack our TMDs-based vdWs heterostructure since interlayer excitons can potentially be formed regardless of the layer orientation in vertically stacked heterostructures. ${ }^{8}$ As shown in Fig. 2d, PL spectroscopy is performed on our SL $\mathrm{MoSe}_{2}$ and $\mathrm{SL} \mathrm{WSe}_{2}$ before and after transfer at varying temperatures, with the $1.88 \mathrm{eV}$ pump excitation at $\approx 2 \mathrm{~mW}$ and with an $\approx 1 \mu \mathrm{m}$ beam spot size. PL spectra show each peak of the $\mathrm{SL} \mathrm{MoSe}_{2}(\approx 1.57 \mathrm{eV})$ and $\mathrm{SL} W \mathrm{WS}_{2}(\approx 1.64 \mathrm{eV})$ are obtained with high quantum efficiency; our trilayer shows two PL peaks captured by a Si detector. With decreasing temperature, both $\mathrm{MoSe}_{2}$ and $\mathrm{WSe}_{2}$ peaks are blue-shifted, consistent with prior studies. ${ }^{20,55-58}$ The interlayer optical transition from the type-II heterojunction becomes dominant at low temperatures. We noticed from the 77 and $4 \mathrm{~K}$ trilayer PL curves in Fig. $2 \mathrm{~d}$ that when the interlayer PL is more dominated, the intralayer $\mathrm{PL}$ is more quenched suggesting an efficient ultrafast charge transfer in the trilayer heterostructure, which agrees with previous ultrafast charge transfer studies of the bilayer heterostructure. ${ }^{48,59,60}$ As shown in the light brown line of Fig. 2d, we observe that the PL intensities of the interlayer excitons can be larger even compared to those of intralayer excitons. (An example comparison of the trilayer to the bilayer is shown in Supplementary Section S3.) It is interesting to note that the addition of a $\mathrm{WSe}_{2}$ layer to the $\mathrm{MoSe}_{2}-\mathrm{WSe}_{2}$ bilayer can result in higher PL quantum yields of both intralayer and interlayer excitons at room temperature and cryogenic temperatures. The reason, as noted in the "Introduction" and "Theoretical analysis" sections, is from the strong wavefunction overlap in the trilayer and additional exciton formation from the extra $\mathrm{WSe}_{2}$ layer 

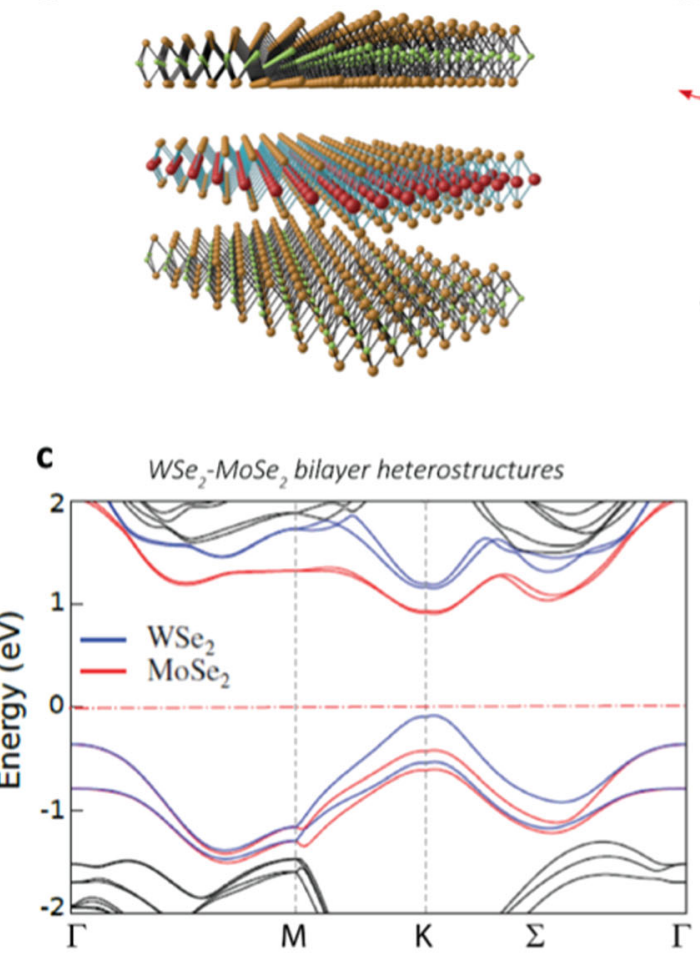

e

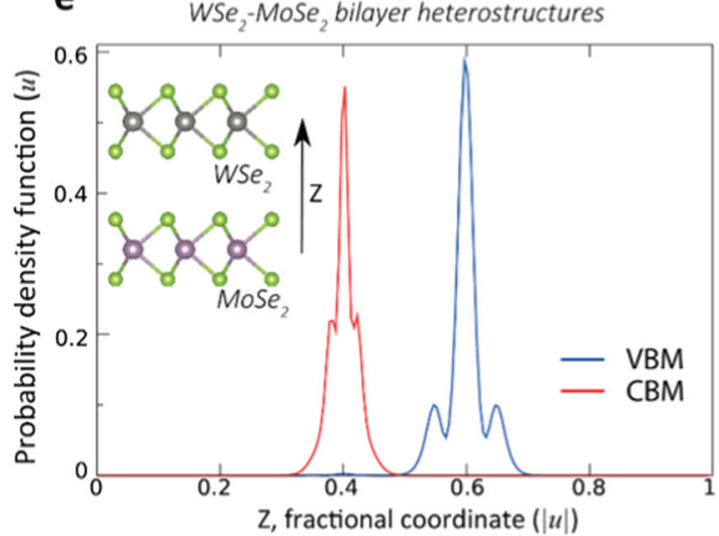

b

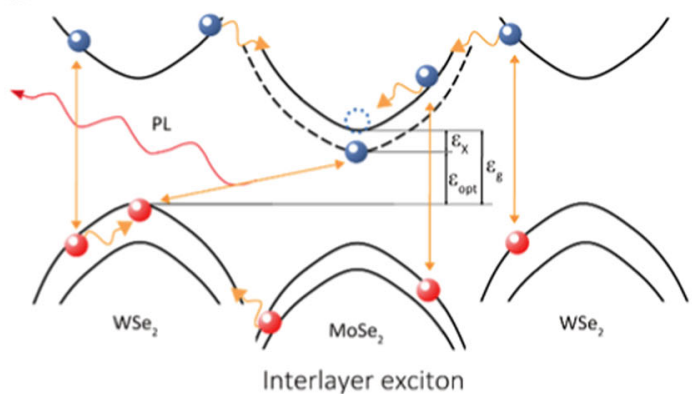

d

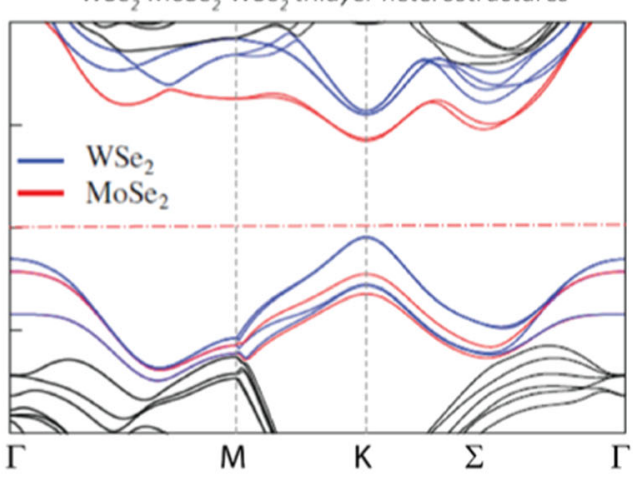

f

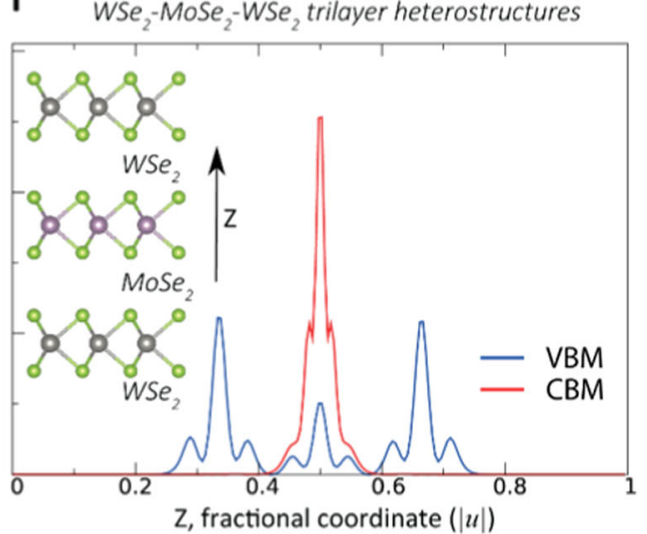

Fig. 1 Excitonic states of TMDs-based trilayer heterostructure, interlayer excitons model, and computed band structure and wavefunction distribution comparisons between the bilayer and trilayer heterostructures. a Illustration of WSe $\mathrm{WoSe}_{2}-\mathrm{WSe}_{2}$ trilayer heterostructure with a sandwiched single layer (SL) MoSe $e_{2}$ between two SLs WSe $e_{2}$. b Schematic summary of the interlayer exciton radiative recombination predicted by type-ll heterojunction. $\varepsilon_{\mathrm{X}}, \varepsilon_{\mathrm{opt}}$, and $\varepsilon_{\mathrm{g}}$ represent the exciton binding energy, optical gap, and electronic gap, respectively. c Computed band structure of the WSe $\mathrm{WoSe}_{2}$ bilayer heterostructure. d Computed band structure of the WSe $-\mathrm{MoSe}_{2}-\mathrm{WSe}_{2}$ trilayer heterostructure. e Computed orbital wavefunction of the $\mathrm{WSe}_{2}-\mathrm{MoSe}_{2}$ bilayer heterostructure valence band maximum (VBM) and conduction band minimum $(C B M)$ at $k$-point as a function of the interlayer distance $z$. $\mathbf{f}$ Computed orbital wavefunction of the WSe $\mathrm{WoSe}_{2}-\mathrm{WSe}_{2}$ trilayer heterostructure

absorbance. We note that the PL enhancement due to absorbance from the extra layer is not more than a factor of 2, while the enhancement measured is an order-of-magnitude larger. This additional enhancement is the contribution of the increased wavefunction overlap.

Interlayer excitons in coupled quantum wells have previously been studied at low temperature in order to avoid thermal quenching of the $\mathrm{PL}$ due to nonradiative processes and electron-hole pairs excited to energies outside the light cone. ${ }^{29,30}$ In Fig. 3, we illustrate the temperature-dependent PL measurements from 4 to $245 \mathrm{~K}$ of our trilayer atomic crystal, in order to examine the interlayer many-body excitonic transitions. Here, we use a liquid-nitrogen-cooled InGaAs photodetector with high responsivity from 900 to $1600 \mathrm{~nm}$. In our trilayer heterostructure, a long lower-energy spectral tail can be observed in the main interlayer exciton PL peak and becomes spectrally distinguishable from the neutral interlayer exciton (IEX $\mathrm{I}_{1}$ ) peak at $4 \mathrm{~K}$ as shown in Fig. 3a (1.96 eV pump excitation). We consequently deconvolved our trilayer interlayer lineshape into two peaks with Lorentzian lineshapes. The higher energy (black line fit) and lower energy (red line fit) peaks are denoted as IEX and IEX $_{2}$, respectively, which we will subsequently attribute to the interlayer neutral excitons and trions (charged exciton) respectively as we will further elaborate below. The inset of Fig. 3a shows the spectroscopy with our onedimensional focal plane array InGaAs detector, with the horizontal axis the spectra and the vertical axis the spatial position across the sample via a scanning mirror. 
a

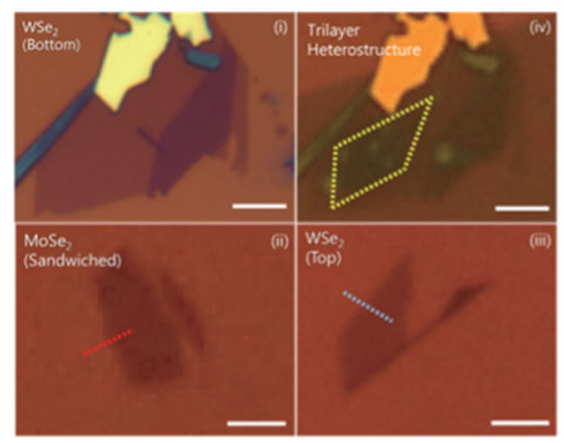

C

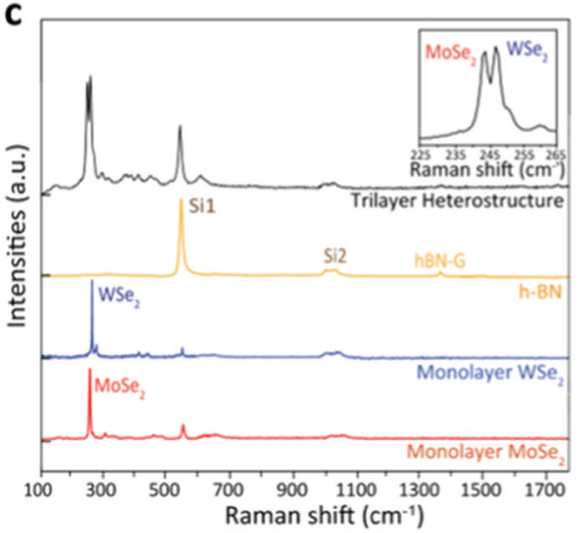

b

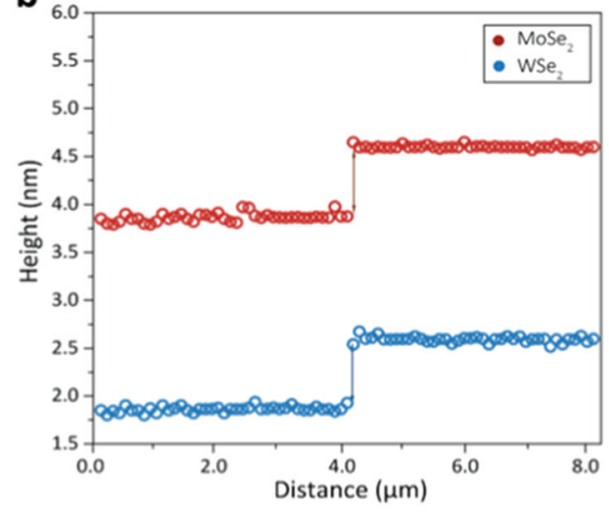

d

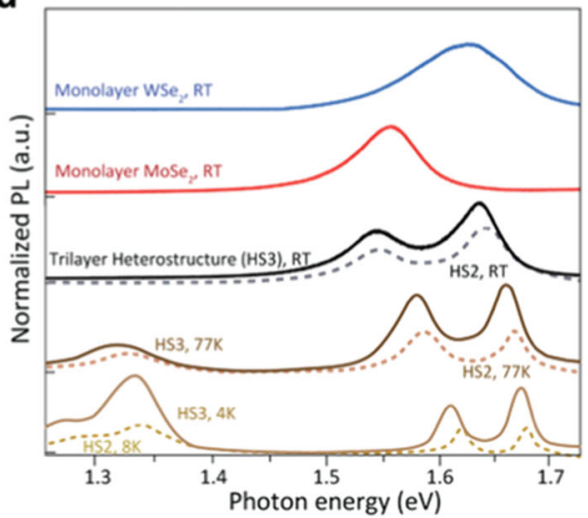

Fig. 2 TMDs-based trilayer van der Waals (vdWs) heterostructure fabrication and characterization. a Microscopic images of the exfoliated transition metal dichalcogenides monolayers [i-iii] and trilayer vdWs heterostructure [iv] on $\mathrm{SiO}_{2}$ substrate. The dashed red and blue lines in the lower panels denote the atomic force microscopy (AFM) regions. The area enclosed by dashed yellow line indicates the trilayer heterostructure region. Scale bar: $5 \mu \mathrm{m}$. b AFM cross-sectional profiles, where each atomic single-layer (SL) is $\approx 7 \AA$. WSe ${ }_{2}$ and MoSe $\mathrm{Mare}_{2}$ the blue and red lines, respectively. c Raman spectra of the trilayer heterostructure versus each SL (MoSe 2 , WSe $2, h$-BN) decomposition, pumped with a $532 \mathrm{~nm}$ laser. Inset: zoom-in of the Raman shift around $245 \mathrm{~cm}^{-1}$. d Room-temperature PL spectra for the SL WSe ${ }_{2}$ SL MoSe 2 , and the trilayer heterostructure, together with the low-temperature PL spectra from the trilayer heterostructure at 77 and $4 \mathrm{~K}$. The excitation is via a $632 \mathrm{~nm}$ HeNe laser. Dotted lines under the trilayer heterostructure PL spectra are the bilayer heterostructure PL spectra, with especially large enhancement of the lower-energy interlayer exciton transition

Figure $3 \mathrm{~b}$ shows the interlayer exciton spectra at different bath temperature, with the black (IEX) and red (IEX) lines tracking the spectral decomposition. It is worth noting that $\mathrm{IEX}_{2}$ becomes distinguishable only at fairly low temperatures in the interlayer exciton lineshape. As shown in Fig. 3b, both peaks are blue-shifted and broadened as the temperature increases and become undetectable above $245 \mathrm{~K}$. Figure $3 \mathrm{c}$ summarizes the measured temperature dependence of their peak positions, quantified by the bandgap variation with temperature. ${ }^{58}$ Linewidth temperature dependence and the phonon-induced bandgap renormalization model is described in Supplementary Sections S4 and S5, respectively. Under the same pump fluence and as shown in Fig. $3 c$, we note that the energy difference between IEX $X_{1}$ and IEX does not change as temperature increases up to $126 \mathrm{~K}$, the temperature after which the IEX $\mathrm{I}_{2}$ peak is too weak to distinguish with certainty. This is consistent with the fact that peak splitting of exciton and trion depends on the pumping fluence, ${ }^{16,61}$ further supported by our power-dependent PL spectra results later in this study.

Figure $3 \mathrm{~d}$, e shows the neutral exciton and trion PL peak intensities and their ratio as a function of temperature. The PL peak intensities decrease with increasing temperature because in, for example, SL MoS 2 it is attributed to thermally excited excitons which escape the light cone. ${ }^{62}$ Our reduced PL intensity with increasing temperature is attributed to the band structure of our trilayer heterostructure, which resembles that of $\mathrm{SL} \mathrm{MoSe}_{2}$ near the band edges despite the existence of two WSe $\mathrm{W}_{2}$ layers. In addition, we note that the phonon-assisted transitions move the weight from the main PL peak to lower energies and thereby reduce the $\mathrm{PL}$ peak intensities. Furthermore, since the promoted nonradiative process competes readily with radiative recombination of the interlayer excitons, the interlayer radiative recombination is not observed at room temperature. In Fig. 3e, we plot the ratio of $\mathrm{PL}$ peak intensities, $I_{\mathrm{IEX} 2} / \mathrm{I}_{\mathrm{IEX} 1}$, which increases from $\approx 0.13$ at $96 \mathrm{~K}$ to $\approx 0.28$ at $4 \mathrm{~K}$ due to suppressed nonradiative recombination and high carrier densities. An abrupt rise around $10 \mathrm{~K}$ is typically induced by a rapidly increasing lifetime of trions and is a key feature of trions. ${ }^{20}$ Discussion about the abrupt rise using mass-action model is detailed in the Supplementary S9. We note that the interlayer radiative recombination channels-in both the neutral exciton and trion-may involve the processes of optical phonons in order to conserve momentum in the band-to-band transition by optical phonon absorption and phonon emission. This is especially the case for the trion which has approximately two times larger full-width half-maximum (FWHM) than the neutral exciton (detailed in Supplementary Figure S4) and has a stronger thermal dependence.

In our experimental measurements, we have assigned the two distinct interlayer PL emission peaks IEX $\mathrm{I}_{1}$ and $\mathrm{IEX}_{2}$ to the neutral exciton and the three-body trion, respectively. More details on the interlayer excitonic radiative recombination pathways are described in Supplementary Section 2 and Figure S2. For the lower energy 
a

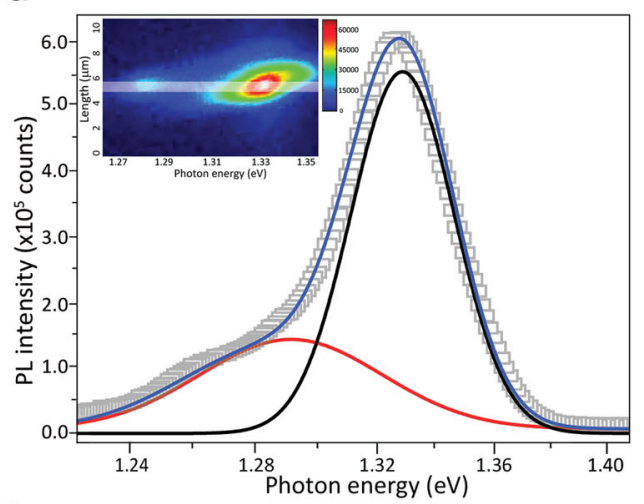

b

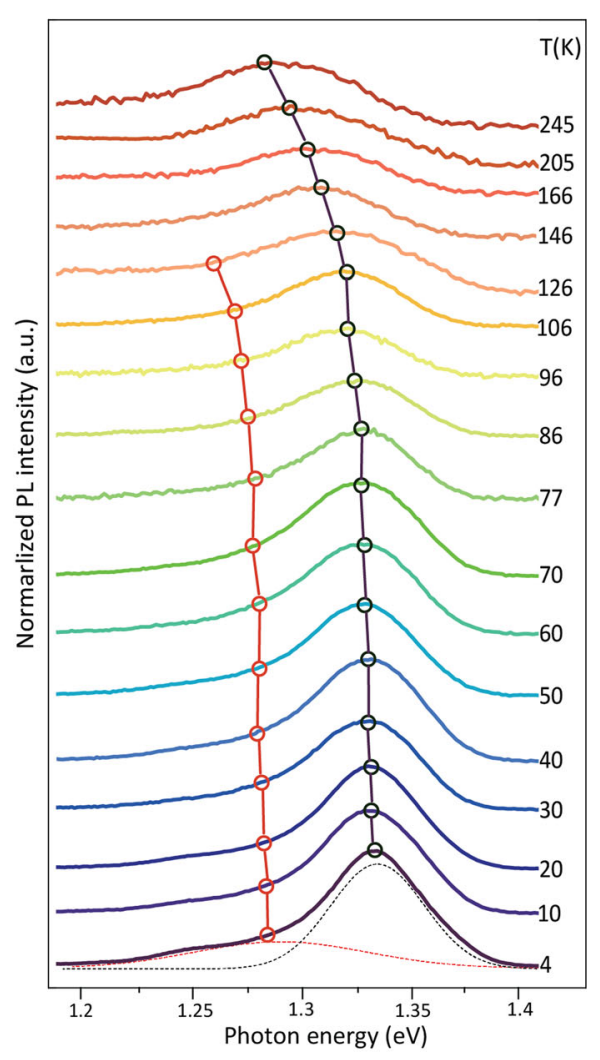

C

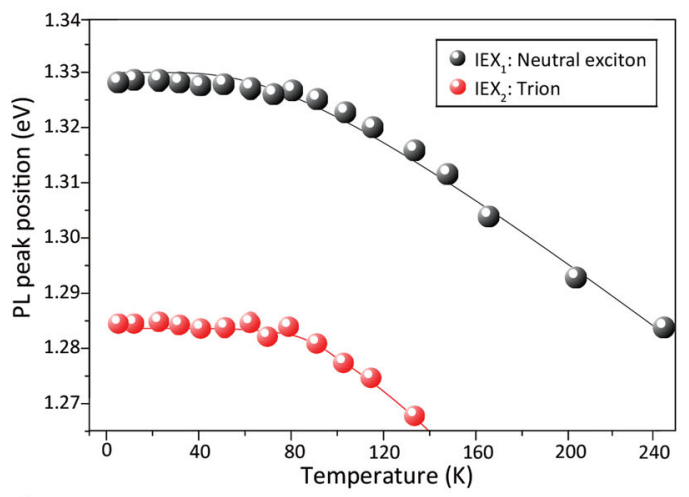

d

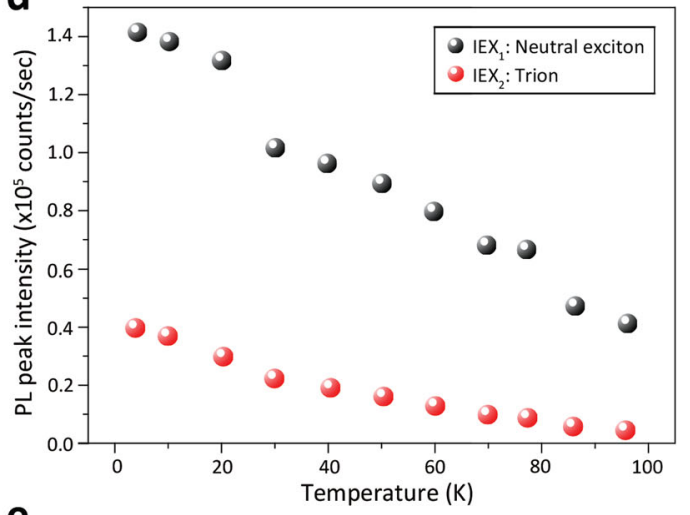

e

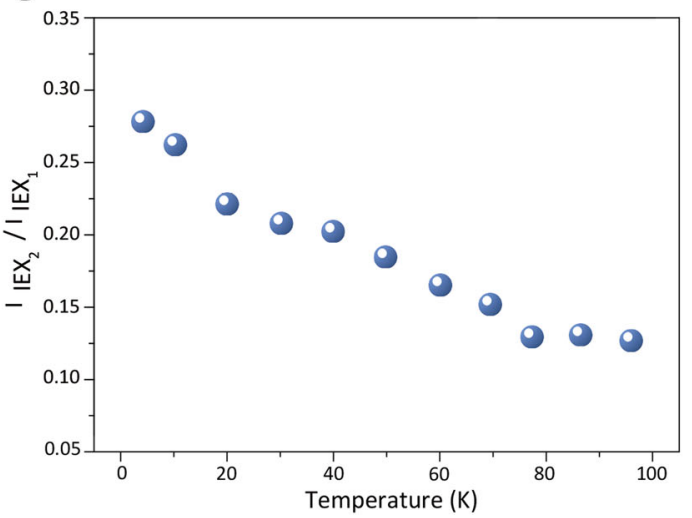

Fig. 3 Temperature-dependent PL intensities and spectral lineshapes of the interlayer excitons in the trilayer heterostructure. a Indirect excitons PL spectra at $4 \mathrm{~K}$, fitted with a blue line. Both IEX $\mathrm{I}_{1}$ : neutral exciton (black) and IEX $\mathrm{E}_{2}$ : trion (red) lines are fitted with Lorentzian lineshapes. Inset image shows PL spectrum captured by a focal plane array detector with horizontal axis the spectra and the vertical axis the spatial position across the sample in one direction. b Temperature-dependent indirect PL spectra in trilayer heterostructure with pump excitation at $1.96 \mathrm{eV}$ and $\approx 1.2 \mathrm{~mW}$ reaching sample. The black and red lines denote the respective peaks, with the dashed black and red curves at the $4 \mathrm{~K}$ spectra illustrating the Lorentzian lineshape decomposition. The data were obtained from the same spot on the sample. The spectra are offset for clarity. c PL peak shifts of the neutral exciton (IEX $)_{1}$ and trion (IEX 2 ) deduced from the main panel and fitted with semiconductor bandgap temperature-dependence model. The phonon-induced bandgap renormalization is described in Supplementary Section S5. d PL peak intensities at different cryogenic temperatures. Black and red dots indicate the PL intensities of neutral exciton and trion, respectively. $\mathbf{e}$ The ratio of neutral exciton (IEX) to trion (IEX $)$ peak intensities in the trilayer heterostructure are illustrated as a function of bath temperature

$\left(\mathrm{IEX} \mathrm{X}_{2}\right)$ interlayer peak, we have also considered two other scenarios but both are unlikely. The first is a phonon-assisted indirect radiative recombination between valence band maximum (VBM) of WSe $\mathrm{W}_{2}$ at $\mathrm{K}$ point and conduction band minimum (CBM) of $\mathrm{MoSe}_{2}$ at $\Sigma$-point, per the band diagram of Fig. 1c, d. However, based on our DFT calculations, the second valley at $\Sigma$-point of $\mathrm{MoSe}_{2} \mathrm{CBM}$ is located at $\approx 200 \mathrm{meV}$ higher than K-point of $\mathrm{MoSe}_{2} \mathrm{CBM}$. This large energy requirement makes it hard for the carriers to thermalize in this phonon-assisted transition, even at room temperature $(\approx 25.7 \mathrm{meV})$. The second considered scenario is that of a potential sub-band above the K-point of $\mathrm{MoSe}_{2}$ CBM. However, in our DFT calculations, we could not find the sub-band or energy splitting at $\mathrm{MoSe}_{2} \mathrm{CBM}$ nor the $\mathrm{WSe}_{2} \mathrm{VBM}$. This further supports that the IEX 2 transition arises from the interlayer trions.

In Fig. 4, we summarize the pump fluence dependence of the two interlayer excitons in our trilayer heterostructure by tuning the optical injection of the carriers. While the spectral shift of interlayer neutral exciton under increasing pump fluence has only a slight blue-shift, the spectral position of the trion is significantly more red-shifted with increasing pump fluence. This is illustrated 

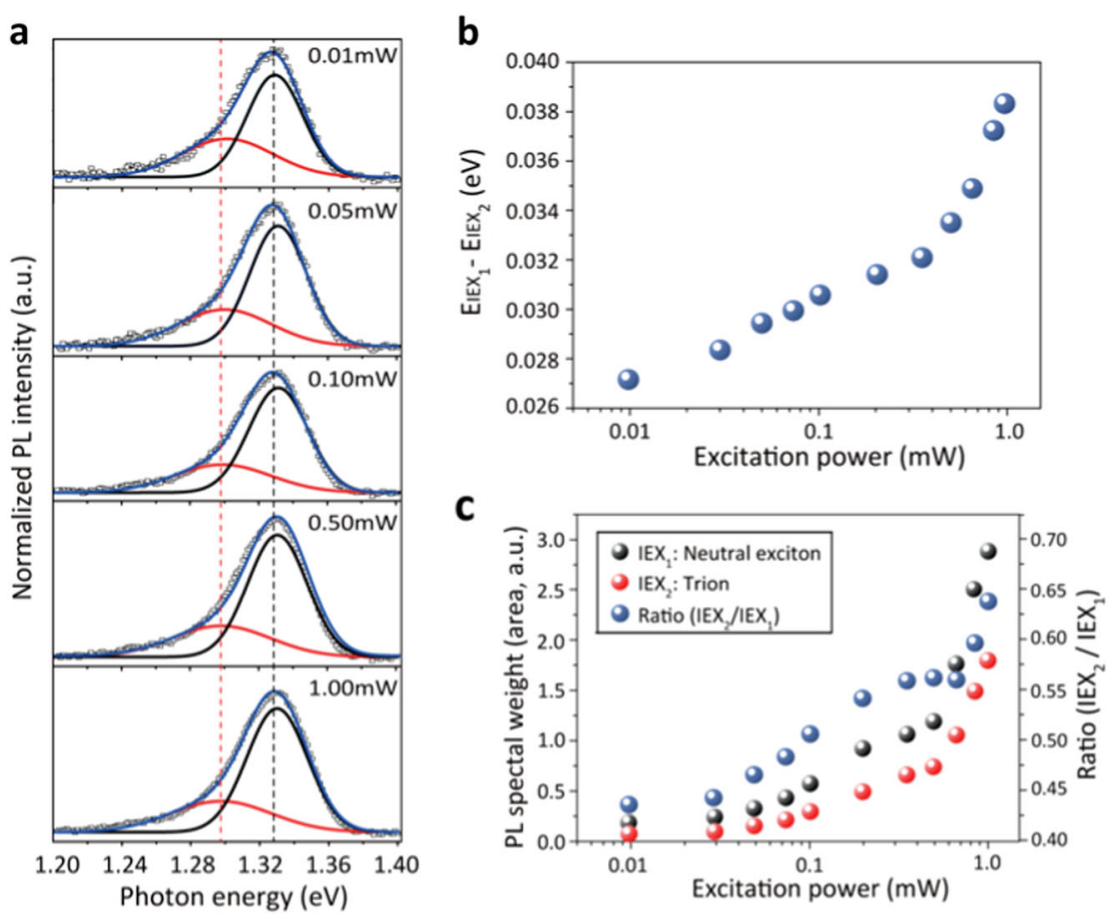

Fig. 4 Power-dependent PL spectra in the trilayer heterostructure at $4 \mathrm{~K}$. a Power-dependent PL spectra with Lorentzian-fitted neutral exciton (black curve) and trion (red curve) at $4 \mathrm{~K}$. b Energy splitting (trion binding energy: $\mathrm{E}_{\mathrm{IEX} 1}-\mathrm{E}_{\mathrm{IEX} 2}$ ) between two interlayer optical transition peaks as a function of excitation power (log scale). c PL spectral weight of neutral exciton (black dots) and trion (red dots), and the ratio of trion to neutral exciton (blue dots), as a function of pump excitation power

in Fig. 4a. Figure 4b summarizes the extracted energy difference between the peaks of the two electronic states for increasing photocarrier injection, illustrating a resulting linear dependence. A description of the two peak energy difference and the trion binding energy, in the presence of carrier screening, is detailed in Supplementary Section S2. Based on this energy splitting, we extract the trion binding energy of $\approx 27 \mathrm{meV}$ from Fig. $4 \mathrm{~b}^{19}{ }^{19}$ which is higher than the thermal activation energy at room temperature and even comparable with intralayer trion binding energy of SL TMDs, ${ }^{16,19-21,58,63}$ and $\approx 28 \mathrm{meV}$ negative charged interlayer exciton binding energy in vertical stack $\mathrm{MoS}_{2} / \mathrm{WS}_{2}$ heterostructures. $^{46}$ This increasing spectral shift and binding energy with photocarrier density is another evidence for the interlayer trions. ${ }^{19,39}$ This is because, with increasing photocarrier injection, free charge requires more energy to occupy the three-body trion excitonic states. In turn, we plot the PL spectral weights of the neutral exciton and trion in Fig. $4 c$ for increasing excitation fluence. With increasing photocarrier injection, the spectral weight and the ratio of the trion to the neutral exciton increases, which is supporting evidence of the interlayer trions existence. ${ }^{39}$

\section{DISCUSSION}

We further investigate the lifetimes of the interlayer neutral exciton and trion, to help elucidate the many-body process. We build the time-resolved photoluminescence (TRPL) setup by means of a time-correlated single-photon counting (TCSPC) system and conduct temperature-, pump fluence-, and pump wavelength-dependent TRPL experiments. All results are fitted biexponentially to the experimental data. Radiative recombination of excitons is difficult to obtain at room temperature because nonradiative recombination is dominant. Accordingly we perform lifetime measurements at cryogenic temperatures, with $\approx 9 \mu \mathrm{J} / \mathrm{cm}^{2}$ pump fluence and $1.88 \mathrm{eV}(660 \mathrm{~nm})$ pump wavelength, above both the SL $\mathrm{MoSe}_{2}$ and SL WSe 2 direct gaps. Figure $5 a, b$ summarizes the recombination lifetimes of interlayer neutral exciton and trion at various cryogenic temperatures. We note that, in the measurement of the IEX 2 lifetimes, we band-pass filter the spectrum with center wavelength around $990 \pm 2 \mathrm{~nm}$ and FWHM of $10 \pm 2 \mathrm{~nm}$, to select the $\mathrm{PL}$ centered $\approx 985 \mathrm{~nm}$ in order to exclude the dynamics of the interlayer neutral exciton. As shown in Fig. $5 \mathrm{a}$, the lifetimes of our trilayer heterostructure interlayer exciton (IEX $)_{1}$ are $\approx 2.54 \mathrm{~ns}(4 \mathrm{~K}), 2.47 \mathrm{~ns}(40 \mathrm{~K}), 1.84 \mathrm{~ns}(80 \mathrm{~K})$, and $0.41 \mathrm{~ns}(120 \mathrm{~K})$, on the same order-of-magnitude as prior bilayer heterostructure studies ${ }^{47}$ and theoretical calculations. ${ }^{32}$ For our trilayer heterostructure interlayer trion $\left(\mathrm{IEX}_{2}\right)$, they are $\approx 1.24 \mathrm{~ns}$ $(4 \mathrm{~K})$ and $0.47 \mathrm{~ns}(20 \mathrm{~K})$. At higher bath temperatures, nonradiative recombination (including phonon-assisted processes) is faster, resulting in the shorter effective lifetimes observed.

Figure $5 c$ summarizes the extracted lifetimes from Fig. $5 a, b$. Although the data points are sparse, we note that the lifetime of the interlayer excitons decreases linearly with increasing temperature and the interlayer trions decreases faster than interlayer excitons. This also correlates with the observed faster trion lifetimes in the finite bath temperature TRPL and the broader linewidths of the trions. We note that the lifetime of the interlayer trion is not observable above $20 \mathrm{~K}$ due to the low photon counts. To further explore the properties of interlayer exciton, we turn our attention to the interlayer exciton lifetimes under various pump fluences and pump wavelengths. As shown in Fig. $5 d$, e, the lifetime of interlayer exciton (IEX $)$ is weakly dependent on carrier injection compared to the bath temperature. As the pump fluence increases, the lifetime only decreases slightly from $\approx 2.72 \mathrm{~ns}$ $\left(1.6 \mu \mathrm{J} / \mathrm{cm}^{2}\right)$ to $\approx 2.53 \mathrm{~ns}\left(8.1 \mu \mathrm{J} / \mathrm{cm}^{2}\right)$. With increasing pump fluence, the interlayer exciton density increases which cause a blue-shift. The dipolar exciton-exciton interaction may lead to the change of peak positions and the decrease of lifetime. ${ }^{64}$ The decrease of lifetime with increasing excitation fluence indicates that residual defect states are involved, as shown in Fig. $5 \mathrm{~d}$ for the $\mathrm{IEX}_{1}$ interlayer lifetime (lifetime of IEX 2 with increasing excitation fluence is not distinctly resolvable). The presence of residual defects also explains why the PL intensities of interlayer exciton 
a

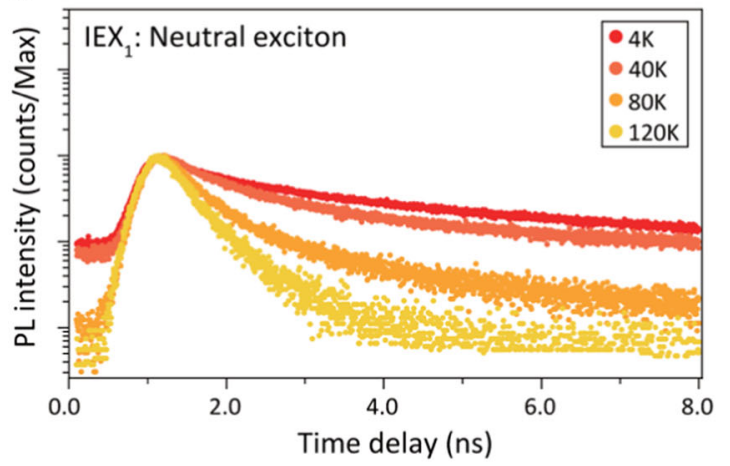

b

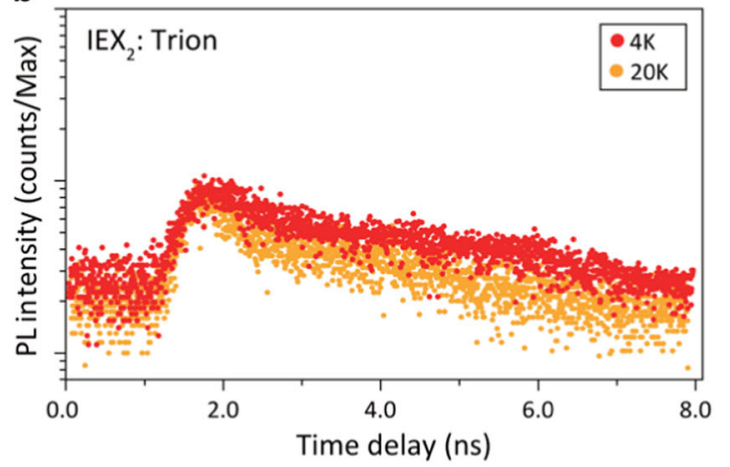

C

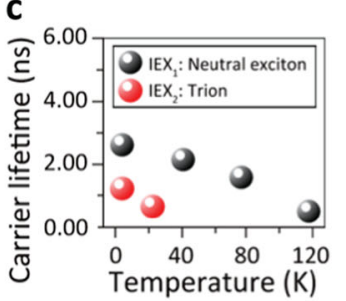

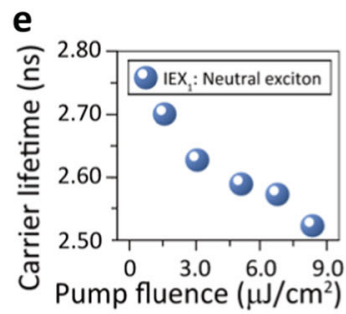

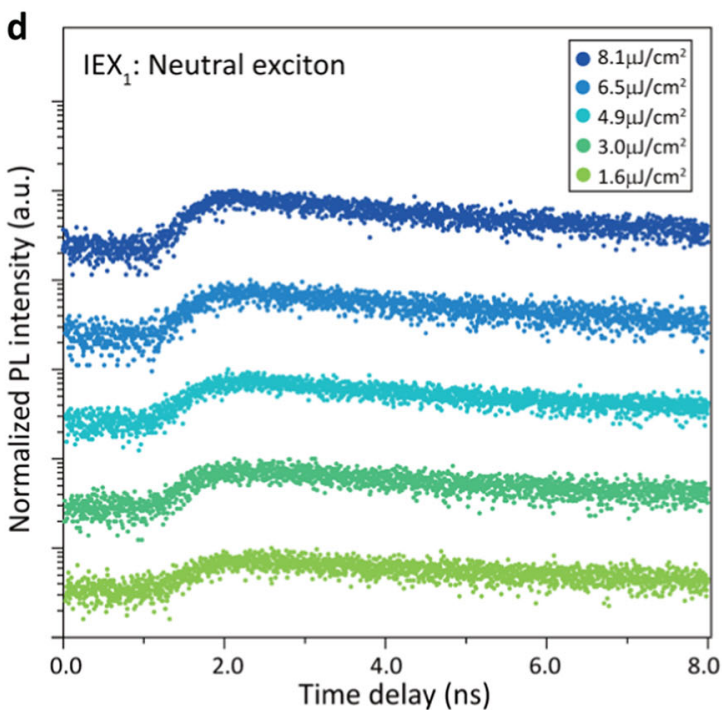

Fig. 5 Interlayer excitons lifetime and its dependence on temperature and photocarrier injection in the trilayer heterostructure. a, b Temperature-dependent carrier lifetimes of interlayer neutral exciton $\left(\mathrm{IEX}_{1}\right)$ and trion $\left(\mathrm{IEX} \mathrm{X}_{2}\right)$ in trilayer heterostructure. Samples are excited with $\approx 9 \mu \mathrm{J} / \mathrm{cm}^{2}$ power fluence at $1.88 \mathrm{eV}(660 \mathrm{~nm})$. c Carrier lifetimes of IEX 1 and IEX 2 with different temperatures at pump fluence of $\approx 9 \mu \mathrm{J} /$ $\mathrm{cm}^{2}$. The carrier relaxations are fitted with exponentials to extract the carrier lifetimes. $\mathbf{d}$ Time-resolved radiative recombination of IEX ${ }_{1}$ under different pump fluences at $4 \mathrm{~K}$. e Carrier lifetimes of IEX ${ }_{1}$ under different pump fluences at $4 \mathrm{~K}$

and trion decrease as temperature increases. We also conduct pump wavelength-resolved TRPL experiment, which is detailed in Supplementary Section S6. There is no correlation between the pump wavelength and the lifetimes of the interlayer exciton. This is because the electron-phonon decay dynamics to the band edge in TMDs is on the order of picoseconds, much shorter than the radiative recombination of interlayer excitons. ${ }^{65-67}$

In this study, we demonstrated the enhanced Coulomb interactions in $\mathrm{WSe}_{2}-\mathrm{MoSe}_{2}-\mathrm{WSe}_{2}$ trilayer vdWs heterostructures, through interlayer radiative recombination of the neutral and charge excitonic states. Designed through spin-polarized DFT with exchange correlation and spin-orbit coupling, we examined typeII heterostructures with increased optical transition oscillator strengths. Fabricated via $h$-BN enabled dry transfer, the trilayer heterostructure exhibits an $\approx 3 \times$ (at $77 \mathrm{~K}$ ) and $5 \times$ (at lower temperature) stronger photoluminescence in our measurements. This arises from the more spatially distributed hybridized valence band state across the trilayer which, while the conduction band is localized only at the $\mathrm{MoSe}_{2}$ layer, enables larger overlap and oscillator strengths. Our cryogenic steady-state and time-resolved photoluminescence measurements elucidate the additional presence of a three-body excitonic state in the interlayer radiative recombination, with the trion binding at $\approx 27 \mathrm{meV}$ and largely dependent on the pump fluence and carrier densities instead of the bath temperature. Conversely, the trilayer interlayer radiative lifetimes are largely dependent on the bath temperature instead of the pump fluence and exciton wavelengths, promoted by phonon-assisted transitions. The relatively long radiative lifetimes measured up to $2.54 \mathrm{~ns}$ at $4 \mathrm{~K}$ enables next-generation excitonic devices. ${ }^{68}$ Future experiments include gating the heterostructures with controlled carrier densities and spectrally separated excitonic resonances to further clarify their roles in exciton dephasing and population relaxation. ${ }^{17,69}$ Also, previous studies about the ultrafast charge transfer between layers within the $\mathrm{MoS}_{2} / \mathrm{WS}_{2}$ heterostructures reveal the fs scale dynamics which can be tuned significantly by changing stacking configuration. ${ }^{53,63,64}$ So future ultrafast pump-probe spectroscopy experiment on our trilayer compared with the bilayer heterostructure can be an interesting topic about the effect of extra $\mathrm{WSe}_{2}$ layer, inducing its stacking configuration, on the charge transfer dynamics. With advanced 2D material growth techniques and computational methods, ${ }^{27,70}$ we believe our trilayer heterostructures support the understanding of interlayer light-matter interactions with facilitated carrier transfer and type-II heterojunctions, paving new ways to tailor the electronic and optoelectronic character of vdWs heterostructures.

\section{METHODS}

Computational method

In the computational modeling of the $\mathrm{MoSe}_{2}$ and $\mathrm{WSe}_{2}$ based heterostructures, the atomic structure of monolayer $\mathrm{MoSe}_{2}$ and $\mathrm{WSe}_{2}$ are first optimized using first-principles pseudopotential calculations based on the spin-polarized DFT within the generalized gradient approximation (GGA). The optimized monolayers are placed on top of each other with proper interlayer distances that correspond to the ground state energy for each heterostructure. During this process, van der Waals corrections are implemented following the DFT-D2 method of Grimme. ${ }^{71}$ Projectoraugmented wave potentials (PAW) are used in the calculations and the exchange-correlation potential is approximated with Perdew-Burke-Ernzerhof functional. ${ }^{72,73}$ During the geometrical optimization steps, the Brillouin zone (BZ) is sampled in the Monkhorst-Pack scheme, where the convergence in energy as a function of the number of $k$-points is tested. The $k$-point sampling of $(21 \times 21 \times 1)$ is found to be 
suitable for the BZ corresponding to the primitive $\mathrm{MoSe}_{2}$ and $\mathrm{WSe}_{2}$ unit cells. The heterostructures are optimized using the conjugate gradient method, where the total energy and atomic forces are minimized. The energy convergence value between two consecutive steps is chosen as $10^{-5} \mathrm{eV}$. A maximum force of $0.01 \mathrm{eV} / \AA$ is allowed on each atom. In order to avoid interactions between periodic unit cells, a supercell with $20 \AA$ vacuum is used. The optimized heterostructures are then used for the electronic band structure calculations. The band diagrams are obtained by calculating the energies at $150 \mathrm{k}$-points between the $\Gamma-\mathrm{M}-\mathrm{K}-\Gamma$ high symmetry points in the BZ. The effects of spin-orbit coupling on the electronic structures are calculated by performing non-collinear computations as implemented in VASP. ${ }^{74}$ The real-space wavefunctions are obtained from the DFT calculations using the binary wavefunction file that is produced by VASP. The binary WAVECAR file that is produced after a series of DFT calculations at the K-point is then mapped by WaveTrans, which correlates the binary wavefunctions with the corresponding lattice points in the reciprocal space and their associated planewave coefficients. The real-space pseudo-wavefunction is constructed again using WaveTrans. ${ }^{75}$

\section{Sample preparation and characterization}

$\mathrm{SL} \mathrm{MoSe}_{2}$ and $\mathrm{SL} \mathrm{WSe}_{2}$ flakes are separately prepared by mechanical exfoliation on clean $290 \mathrm{~nm} \mathrm{SiO} / 2 / \mathrm{Si}$ substrates. A thin layer of hexagonal boron nitride is first exfoliated on a PMMA/PPC stack, which is then used for layer-by-layer picking up of the prepared $\mathrm{WSe}_{2}$ and $\mathrm{MoSe}_{2}$ in order. The picked up $\mathrm{MoSe}_{2} / \mathrm{WSe}_{2} / h$-BN/polymer stack is finally transferred on a $\mathrm{WSe}_{2} / \mathrm{SiO}_{2} / \mathrm{Si}$ substrate. ${ }^{25,26}$ During the entire dry transfer process, no solvent is involved to ensure the atomically clean interface between the $2 \mathrm{D}$ materials. Raman measurement (Renishaw, inVia basis) is conducted using a $532 \mathrm{~nm}$ laser to map out the sample quality.

\section{Cryogenic micro-photoluminescence and time-resolved photoluminescence}

We use an optical cryostat (Janis Research ST-100) to measure the cryogenic photoluminescence at $4 \mathrm{~K}$. Measurements are carried out using a $\mathrm{Si}$ detector (Newport Model 2151) for the shorter wavelength regime $(400-1000 \mathrm{~nm})$ with a continuous-wave $\mathrm{He}-\mathrm{Ne} 632.8 \mathrm{~nm}$ pump laser. For the longer wavelengths $(900-1600 \mathrm{~nm})$ where interlayer exciton and trion can exist, an InGaAs focal plane array detector with liquid nitrogen cooling system (Princeton Instruments 2D-OMA) is used with high responsitivity. The detector signal is improved by using a lock-in amplifier and selectively filtered by a spectrometer (Princeton Instruments SpectraPro 2500i). TRPL measurements are performed using a TCSPC system with PicoQuant. The sample is excited with a $30 \mathrm{ps} 39 / 78 \mathrm{MHz}$ tunable pulse laser system (NKT Photonics SuperK EXTREME EXW-12) and detected with a single-photon counting module (SPCM-AQR-16) attached to a monochromator and processed by a PicoHarp 300 correlating system with $\approx 300$ ps resolution. The TRPL measurements are conducted at low temperature with optical filters in an enclosed optical setup to prevent pump and stray ambient light from affecting the detectors.

\section{DATA AVAILABILITY}

All relevant data are available upon a reasonable request from corresponding authors.

\section{ACKNOWLEDGEMENTS}

The authors are grateful for helpful discussions with Philip Kim, Tony van Buuren, Jinkang Lim, Zhangji Zhao, Jin Ho Kang, Baicheng Yao, and Yandong Luo. We acknowledge support from the University of California-National Laboratory Research Program and the National Science Foundation (DMR-1611598).

\section{AUTHOR CONTRIBUTIONS}

C.C. designed this work. C.C., H.K., and J.H. performed the optical measurements and data analysis. H.-C.C. and C.C. led the device fabrication. C.C., J.C., S.-H.B., and X.D. provided TMDs materials and sample characterizations. C.C., A.K.V., S.-W.H., V.O.Ö., R. G., K.K., T.L., and C.W.W. contributed to the theoretical analysis and simulations. C.W. W. supported and supervised the research. C.C., K.K., T.L., and C.W.W. prepared the manuscript.

\section{ADDITIONAL INFORMATION}

Supplementary information accompanies the paper on the npj 2D Materials and Applications website (https://doi.org/10.1038/s41699-018-0075-1).

Competing interests: The authors declare no competing interests.

Publisher's note: Springer Nature remains neutral with regard to jurisdictional claims in published maps and institutional affiliations.

\section{REFERENCES}

1. Amani, M. et al. Near-unity photoluminescence quantum yield in $\mathrm{MoS}_{2}$. Science 350, 1065-1068 (2015).

2. Lin, Y. et al. Dielectric screening of excitons and trions in single-layer $\mathrm{MoS}_{2}$. Nano Lett. 14, 5569-5576 (2014).

3. Kato, Y. K., Myers, R. C., Gossard, A. C. \& Awschalom, D. D. Observation of the spin hall effect in semiconductors. Science 306, 1910-1913 (2004).

4. Xu, X., Wang, Y., Di, X. \& Heinz, T. F. Spin and pseudospins in layered transition metal dichalcogenides. Nat. Phys. 10, 343-350 (2014).

5. Mak, K. F., He, K., Shan, J. \& Heinz, T. F. Control of valley polarization in monolayer $\mathrm{MoS}_{2}$ by optical helicity. Nat. Nanotechnol. 7, 494-498 (2012).

6. Wang, H. et al. Integrated circuits based on bilayer $\mathrm{MoS}_{2}$ transistors. Nano Lett. 12, 4674-4680 (2012).

7. Low, T. et al. Polaritons in layered 2D materials. Nat. Mater. 16, 182-194 (2016).

8. Chen, $\mathrm{H}$. et al. Ultrafast formation of interlayer hot excitons in atomically thin $\mathrm{MoS}_{2} / \mathrm{WS}_{2}$ heterostructures. Nat. Commun. 7, 12512 (2016).

9. Zhu, W. et al. Electronic transport and device prospects of monolayer molybdenum disulphide grown by chemical vapour deposition. Nat. Commun. 5, 3087 (2014).

10. Sim, S. et al. Selectively tunable optical Stark effect of anisotropic excitons in atomically thin $\operatorname{ReS}_{2}$. Nat. Commun. 7, 13569 (2016).

11. Cha, S. et al. 1s-intraexcitonic dynamics in monolayer $\mathrm{MoS}_{2}$ probed by ultrafast mid-infrared spectroscopy. Nat. Commun. 7, 10768 (2016).

12. Radisavljevic, B. et al. Single-layer $\mathrm{MoS}_{2}$ transistors. Nat. Nanotechnol. 6, 147-150 (2011).

13. Wang, Q. H., Kalantar-Zadeh, K., Kis, A., Coleman, J. N. \& Strano, M. S. Electronics and optoelectronics of two-dimensional transition metal dichalcogenides. Nat. Nanotechnol. 7, 699-712 (2012).

14. Yuan, L. \& Huang, L. Exciton dynamics and annihilation in $W_{2} 2 D$ semiconductors. Nanoscale 7, 7402-7408 (2015).

15. He, K. et al. Tightly bound excitons in monolayer WSe $\mathrm{W}_{2}$. Phys. Rev. Lett. 113, 026803 (2014)

16. Mak, K. F. et al. Tightly bound trions in monolayer $\mathrm{MoS}_{2}$. Nat. Mater. 12, 207-211 (2013).

17. Sie, E. J. et al. Large, valley-exclusive Bloch-Siegert shift in monolayer $\mathrm{WS}_{2}$. Science 355, 1066-1069 (2017).

18. Jones, A. M. et al. Excitonic luminescence upconversion in a two-dimensional semiconductor. Nat. Phys. 12, 323-327 (2015).

19. Jones, A. M. et al. Optical generation of excitonic valley coherence in monolayer WSe $e_{2}$. Nat. Nanotechnol. 8, 634-638 (2013).

20. Ross, J. S. et al. Electrical control of neutral and charged excitons in a monolayer semiconductor. Nat. Commun. 4, 1474 (2013).

21. Aivazian, G. et al. Magnetic control of valley pseudospin in monolayer WSe $\mathrm{W}_{2}$. Nat. Phys. 11, 148-152 (2014).

22. You, Y. et al. Observation of biexcitons in monolayer WSe 2 . Nat. Phys. 11, 477-481 (2015).

23. Kim, J. et al. Layer-resolved graphene transfer via engineered strain layers. Science 342, 833-836 (2013).

24. Bae, S.-H. et al. Unveiling the carrier transport mechanism in epitaxial graphene for forming wafer-scale, single-domain graphene. Proc. Natl. Acad. Sci. U.S.A. 114, 4082-4086 (2017)

25. Liu, Y. et al. Toward barrier free contact to molybdenum disulfide using graphene electrodes. Nano Lett. 15, 3030-3034 (2015).

26. Cheng, H. C. et al. Van der Waals heterojunction devices based on organohalide perovskites and two-dimensional materials. Nano Lett. 16, 367-373 (2016).

27. Li, M.-Y. et al. Epitaxial growth of a monolayer $\mathrm{WSe}_{2}-\mathrm{MoS}_{2}$ lateral $p-n$ junction with an atomically sharp interface. Science 349, 524-528 (2015).

28. Kim, Y. et al. Remote epitaxy through graphene enables two dimensional material-based layer transfer. Nature 544, 340-343 (2017).

29. Butov, L. V., Zrenner, A., Abstreiter, G., Bohm, G. \& Weimann, G. Condensation of indirect excitons in coupled AlAs/GaAs quantum wells. Phys. Rev. Lett. 73, 304-307 (1994).

30. Butov, L., Imamoglu, A., Mintsev, A., Campman, K. \& Gossard, A. Photoluminescence kinetics of indirect excitons in $\mathrm{GaAs} / \mathrm{Al}_{\mathrm{x}} \mathrm{Ga}_{1-\mathrm{x}} \mathrm{As}$ coupled quantum wells. Phys. Rev. B 59, 1625-1628 (1999). 
31. Rivera, P. et al. Valley-polarized exciton dynamics in a $2 \mathrm{D}$ semiconductor heterostructure. Science 35, 668-690 (2016).

32. Rivera, $\mathrm{P}$. et al. Observation of long-lived interlayer excitons in monolayer $\mathrm{MoSe}_{2}-\mathrm{WSe}_{2}$ heterostructures. Nat. Commun. 6, 6242 (2015).

33. Jones, A. M. et al. Spin-layer locking effects in optical orientation of exciton spin in bilayer WSe $\mathrm{Wat}_{2}$. Phys. 10, 130-134 (2014).

34. Gong, C. et al. Band alignment of two-dimensional transition metal dichalcogenides: application in tunnel field effect transistors. Appl. Phys. Lett. 103, 053513 (2013).

35. Özçelik, V. O., Azadani, J. G., Yang, C., Koester, S. J. \& Low, T. Band alignment of two dimensional semiconductors for designing heterostructures with momentum space matching. Phys. Rev. B 94, 035125 (2016).

36. Mak, K. F. \& Shan, J. Photonics and optoelectronics of 2D semiconductor transition metal dichalcogenides. Nat. Photonics 10, 216-226 (2016).

37. Palummo, M., Bernardi, M. \& Grossman, J. C. Exciton radiative lifetimes in two dimensionl transition metal dichalcogenides. Nano Lett. 15, 2794-2800 (2015).

38. Chiu, M.-H. et al. Determination of band alignment in the single layer $\mathrm{MoS}_{2} / \mathrm{WSe}_{2}$ heterojunction. Nat. Commun. 6, 7666 (2015).

39. Bellus, M. Z., Ceballos, F., Chiu, H. \& Zhao, H. Tightly bound trions in transition metal dichalcogenide heterostructures. ACS Nano 9, 6459-6464 (2015).

40. Chiu, M. H. et al. Spectroscopic signatures for interlayer coupling in $\mathrm{MoS}_{2}-\mathrm{WSe}_{2}$ van der Waals stacking. ACS Nano 8, 9649-9656 (2014).

41. Zhang, J. et al. Observation of strong interlayer coupling in $\mathrm{MoS}_{2} / \mathrm{WS}_{2}$ heterostructures. Adv. Mater. 28, 1950-1956 (2016).

42. Hill, H. M., Rigosi, A. F., Rim, K. T., Flynn, G. W. \& Heinz, T. F. Band alignment in $\mathrm{MoS}_{2} / \mathrm{WS}_{2}$ transition metal dichalcogenide heterostructures probed by scanning tunneling microscopy and spectroscopy. Nano Lett. 16, 4831-4837 (2016).

43. Nagler, P. et al. Giant magnetic splitting inducing near-unity valley polarization in van der Waals heterostructures. Nat. Commun. 8, 1551 (2017).

44. Baranowski, M. et al. Probing the interlayer exciton physics in a $\mathrm{MoS}_{2} / \mathrm{MoSe}_{2} /$ $\mathrm{MoS}_{2}$ van der Waals heterostructure. Nano Lett. 17, 6360-6365 (2017).

45. Miller, B. et al. Long-lived direct and indirect interlayer excitons in van der Waals heterostructures. Nano Lett. 17, 5229-5237 (2017).

46. Thorsten, D. \& Kristian, S. T. Interlayer trions in the $\mathrm{MoS}_{2} / \mathrm{WS}_{2}$ van der Waals heterostructure. Nano Lett. 18, 1460-1465 (2018).

47. Rigosi, A. F., Hill, H. M., Li, Y., Chernikov, A. \& Heinz, T. F. Probing interlayer interactions in transition metal dichalcogenide heterostructures by optical spectroscopy: $\mathrm{MoS}_{2} / \mathrm{WS}_{2}$ and $\mathrm{MoSe}_{2} / \mathrm{WSe}_{2}$. Nano Lett. 15, 5033-5038 (2015).

48. Hong, X. et al. Ultrafast charge transfer in atomically thin $\mathrm{MoS}_{2} / \mathrm{WS}_{2}$ heterostructures. Nat. Nanotechnol. 9, 682-686 (2014).

49. Kozawa, D. et al. Evidence for fast interlayer energy transfer in $\mathrm{MoSe}_{2} / \mathrm{WS}_{2}$ heterostructures. Nano Lett. 16, 4087-4093 (2016).

50. Wang, Y., Wang, Z., Yao, W., Liu, G. B. \& Yu, H. Interlayer coupling in commensurate and incommensurate bilayer structures of transition-metal dichalcogenides. Phys. Rev. B 95, 115429 (2017).

51. Kim, J. et al. Observation of ultralong valley lifetime in $\mathrm{WSe}_{2} / \mathrm{MoS}_{2}$ heterostructures. Sci. Adv. 3, e1700518 (2016).

52. Robert, $C$. et al. Exciton radiative lifetime in transition metal dichalcogenide monolayers. Phys. Rev. B 93, 205423 (2016).

53. Singh, A. et al. Trion formation dynamics in monolayer transition metal dichalcogenides. Phys. Rev. B 93, 041401 (2016).

54. Dean, C. R. et al. Boron nitride substrates for high-quality graphene electronics. Nat. Nanotechnol. 5, 722-726 (2010).

55. Wang, G. et al. Valley dynamics probed through charged and neutral exciton emission in monolayer WSe ${ }_{2}$. Phys. Rev. B 90, 075413 (2014).

56. Molina-Sánchez, A., Palummo, M., Marini, A. \& Wirtz, L. Temperature-dependent excitonic effects in the optical properties of single-layer MoS 2 . Phys. Rev. B 93 , 155435 (2016)
57. Zhang, S. et al. Defect structure of localized excitons in a WSe $\mathrm{W}_{2}$ monolayer. Phys. Rev. Lett. 119, 046101 (2017).

58. O'Donnell, K. P. \& Chen, X. Temperature dependence of semiconductor band gaps. Appl. Phys. Lett. 58, 2924-2926 (1991).

59. Ji, Z. et al. Robust stacking-independent ultrafast charge transfer in $\mathrm{MoS}_{2} / \mathrm{WS}_{2}$ bilayers. ACS Nano 11, 12020-12026 (2017).

60. Zhang, J. et al. Interlayer-state-coupling dependent ultrafast charge transfer in $\mathrm{MoS}_{2} / \mathrm{WS}_{2}$ bilayers. Adv. Sci. 4, 1700086 (2017).

61. Zhang, C., Wang, H., Chan, W., Manolatou, C. \& Rana, F. Absorption of light by excitons and trions in monolayers of metal dichalcogenide $\mathrm{MoS}_{2}$ : experiments and theory. Phys. Rev. B 89, 205436 (2014).

62. Withers, F. et al. WSe $\mathrm{W}_{2}$ light-emitting tunneling transistors with enhanced brightness at room temperature. Nano Lett. 15, 8223-8228 (2015).

63. Berkelbach, T. C., Hybertsen, M. S. \& Reichman, D. R. Theory of neutral and charged excitons in monolayer transition metal dichalcogenides. Phys. Rev. B 88, 045318 (2013).

64. Nagler, P. et al. Interlayer exciton dynamics in a dichalcogenide monolayer heterostructure. 2D Mater. 4, 025112 (2017)

65. Wang, H., Zhang, C. \& Rana, F. Ultrafast dynamics of defect-assisted electron-hole recombination in monolayer $\mathrm{MoS}_{2}$. Nano Lett. 15, 339-345 (2015).

66. Lui, C. H. et al. Observation of interlayer phonon modes in van der Waals heterostructures. Phys. Rev. B 91, 165403 (2015).

67. $\mathrm{Yu}, \mathrm{Y}$. et al. Equally efficient interlayer exciton relaxation and improved absorption in epitaxial and nonepitaxial $\mathrm{MoS}_{2} / \mathrm{WS}_{2}$ heterostructures. Nano Lett. 15 486-491 (2015).

68. Grosso, G. et al. Excitonic switches operating at around 100 K. Nat. Photonics 3 , 577-580 (2010)

69. Kim, J. et al. Electrical control of optical plasmon resonance with graphene. Nano Lett. 12, 5598-5602 (2012).

70. Latini, S., Winther, K. T., Olsen, T. \& Thygesen, K. S. Interlayer excitons and band alignment in $\mathrm{MoS}_{2} / \mathrm{hBN} / \mathrm{WSe}_{2}$ van der Waals heterostructures. Nano Lett. 17, 938-945 (2017)

71. Grimme, S. Semiempirical GGA-type density functional constructed with a longrange dispersion correction. J. Comput. Chem. 27, 1787-1799 (2006).

72. Blöch, P. E. Projector augmented-wave method. Phys. Rev. B 50, 17953 (1994).

73. Perdew, J. P., Burke, K. \& Ernzerhof, M. Generalized gradient approximation made simple. Phys. Rev. Lett. 77, 3865-3868 (1996).

74. Kresse, G. \& Furthmüller, J. Efficient iterative schemes for ab initio total-energy calculations using a plane-wave basis set. Phys. Rev. B 54, 11169 (1996)

75. Feenstra, R. M. et al. Low-energy electron reflectivity from graphene. Phys. Rev. $B$ 87, 041406 (2013).

Open Access This article is licensed under a Creative Common cc Attribution 4.0 International License, which permits use, sharing adaptation, distribution and reproduction in any medium or format, as long as you give appropriate credit to the original author(s) and the source, provide a link to the Creative Commons license, and indicate if changes were made. The images or other third party material in this article are included in the article's Creative Commons license, unless indicated otherwise in a credit line to the material. If material is not included in the article's Creative Commons license and your intended use is not permitted by statutory regulation or exceeds the permitted use, you will need to obtain permission directly from the copyright holder. To view a copy of this license, visit http://creativecommons. org/licenses/by/4.0/.

(c) The Author(s) 2018 